\title{
TTR
}

Traduction, terminologie, re?daction

\section{Organisation conceptuelle du domaine et structure de dictionnaire - L'exemple du commerce de détail}

\section{Jeanne Dancette}

Volume 8, numéro 2, 2e semestre 1995

Technolectes et dictionnaires

URI : https://id.erudit.org/iderudit/037221ar

DOI : https://doi.org/10.7202/037221ar

Aller au sommaire du numéro

Éditeur(s)

Association canadienne de traductologie

ISSN

0835-8443 (imprimé)

1708-2188 (numérique)

Découvrir la revue

Citer cet article

Dancette, J. (1995). Organisation conceptuelle du domaine et structure de dictionnaire - L'exemple du commerce de détail. TTR, 8(2), 151-174.

https://doi.org/10.7202/037221ar

\section{Résumé de l'article}

Organisation conceptuelle du domaine et structure de dictionnaire - L'exemple du commerce de détail - Le présent article a pour objectif de présenter un dictionnaire spécialisé bilingue (anglais-français) original. Il est conçu pour répondre aux besoins des traducteurs et les stratégies lexicographiques mises en oeuvre s'orientent autour de trois axes : richesse des propositions d'équivalent, intention et extension des définitions, explicitation des relations sémantiques (ou internotionnelles). L'article propose une discussion de ces axes en fonction de plusieurs paramètres liés au domaine de spécialité : nature et dynamique de la langue commerciale dans le domaine de la distribution, degré d'avancement de la recherche terminologique dans ce domaine, besoin des traducteurs dans cette spécialité. Les grandes lignes théoriques de l'article s'articulent autour de la mise en évidence des structures de connaissances propres au domaine et de la priorité accordée à la démarche cognitive des traducteurs (qui opèrent en discours plutôt qu'en langue).
Tous droits réservés @ C TTR: traduction, terminologie, rédaction — Les auteurs, 1995
Ce document est protégé par la loi sur le droit d'auteur. L’utilisation des services d'Érudit (y compris la reproduction) est assujettie à sa politique d'utilisation que vous pouvez consulter en ligne.

https://apropos.erudit.org/fr/usagers/politique-dutilisation/ 


\section{Organisation conceptuelle du domaine et structure de dictionnaire - L'exemple du commerce de détail $^{1}$}

\section{Jeanne Dancette}

En traduction spécialisée, une des conditions essentielles à la pertinence de l'information terminologique est qu'elle s'articule sur les connaissances thématiques auxquelles les termes renvoient, facilitant ainsi la compréhension du domaine visé. Nous tâcherons, dans le présent article, de démontrer que cette exigence peut être, au moins en partie, satisfaite. C'est en renforçant la dimension cognitive et en la rendant explicite dans le dictionnaire que s'ouvre l'une des voies les plus intéressantes de la terminologie bilingue.

Le dictionnaire bilingue (anglais - français) du commerce de détail que nous sommes en train d'élaborer avec une équipe d'étudiants chercheurs de linguistique et de traduction est la convergence de plusieurs exigences. Pour être un outil terminologique fiable pour le traducteur, il doit offrir de nombreuses possibilités d'équivalents et expliquer clairement les critères de sélection des équivalents. Dictionnaire de langue, il doit contenir les informations linguistiques généralement attendues dans ces ouvrages (description grammaticale et

1. Cette recherche est rendue possible grâce à une subvention du FCAR. Nous exprimons notre gratitude à cet organisme subventionnaire ainsi qu'à notre collaborateur Christophe Réthoré qui a participé à l'analyse des données présentées dans l'article et à Zélie Guével dont les commentaires nous ont permis de préciser notre pensée. 
sémantique, dérivations, usages discursifs, exemples). Enfin, ouvrage thématique, il doit être résolument axé sur la description des concepts et la présentation des connaissances encyclopédiques.

La recherche combinée de ces trois objectifs n'est, certes, pas nouvelle (Sager, 1990; Bessé, 1992, etc.). Par contre, la réalisation en est plus problématique et chaque fois originale. Elle doit tenir compte des spécificités du domaine de spécialité visé, du statut lexicographique relatif au domaine, et aussi des besoins des utilisateurs auquel l'ouvrage est destiné. La discussion proposée dans cet article porte donc sur la concrétisation de ces objectifs en fonction des trois paramètres suivants: a) nature et dynamique de la langue de spécialisation à l'étude, ici le langage du commerce de détail; b) degré d'avancement de la recherche terminologique dans le domaine de spécialisation retenu; c) besoins de la traduction dans les langues de spécialité. Après avoir annoncé le cadre théorique de cette discussion, nous analyserons ces paramètres et présenterons quelques entrées du dictionnaire afin d'illustrer notre approchedictionnairique/terminographiqueet de mieux faire comprendre les difficultés rencontrées.

\section{Cadre théorique et méthodologique}

Quelques points essentiels résument la problématique. Reconnaissant la position particulière du traducteur dans le processus terminologique, le dictionnaire est axé, d'une part, sur la mise en évidence des structures de connaissances, dans une perspective ouverte et évolutive; d'autre part, il reconnaît et valorise la fonction terminologique - éventuellement normalisatrice - du traducteur.

$\mathrm{Au}$ plan pragmatique, le traducteur occupe une position particulière dans le rapport langue-concept. Souvent très éloigné du lieu de production ou du lieu où se négocient les affaires, le traducteur n'est pas un intervenant direct dans la production ou l'échange. Il ne participe donc pas à la culture intuitive des faits. Mais, s'il n'intervient pas dans le processus de production ou d'échange, il participe entièrement à la culture des termes. De par sa fonction de communicateur, il est souvent le premier divulgateur du concept et le premier diffuseur des usages lexicaux. Il est donc activement engagé dans le processus terminologique. À cette responsabilité terminologique doit correspondre 
un haut degré de compréhension des concepts, souvent aussi élevé que celui des spécialistes du domaine. L'ouvrage dictionnairique doit alors faciliter la convergence de ces deux types de savoir.

Cette position particulière entraîne un réalignement théorique. Une des caractéristiquesprincipales du dictionnaire devient alors la mise en évidence des structures de connaissances propres au domaine de spécialité traité. Cette caractéristique se manifeste, dans le dictionnaire, par le traitement systématique des termes et des relations conceptuelles qui les lient entre eux, faisant apparaître l'arbre de domaine, ou, du moins, un ensemble de réseaux conceptuels. Cette interaction entre la cognition et la dénomination est essentielle, tant dans la démarche du traducteur que dans celle du terminologue. Citons Sager et Kageura (1994-1995, p. 138):

Cognition is concerned with ordering items of our perception and experience, which implies relating and comparing these items in order to classify or structure them into concepts and concept classes. Our perception initially discovers similarities among items which it associates with, and groups into the extension of a concept, reserving for the intension of a concept those elements which have motivated our grouping of items in the first place. In a simplified manner we could also say that perception is a process which maps extensional items to provisionally established and intensionally defined concepts.

Notons qu'il s'agit là d'une démarche cognitive, d'un processus, et non pas d'un savoir arrêté et définitif qui se traduirait dans le dictionnaire par des structures de connaissances relativement rigides, faisant l'objet d'un consensus durable. Dans les domaines en évolution rapide, en particulier, il paraît indispensable que le dictionnaire reflète les fluctuations terminologiques et conceptuelles du domaine. C'est notamment le cas dans le commerce de détail dont il est question dans le présent article.

Les conséquences théoriques doivent être mentionnées. À la différence des dictionnaires habituels, le dictionnaire du commerce de détail ne prétend aucunement être la réflexion exacte de la langue, au sens saussurien, dans ses aspects lexicaux (ou plus exactement d'un langage dans le cas présent) ni offrir un inventaire fini de termes. Il $s$ 'inscrit plutôt dans une perspective évolutive. Les termes sont présentés 
et définis en contexte, au moyen parfois d'illustrations et d'exemples concrets quand cela précise la description du concept. Les équivalents et les informations linguistisques ne sont pas donnés dans une optique prescriptive ou normative, mais plutôt comme des suggestions afin d'aider l'utilisateur (le traducteur) à comprendre le sens, à instaurer des usages, éventuellement même à enrichir et mettre à jour le dictionnaire là où il est incomplet, quant aux équivalents interlinguistiques ou à l'information conceptuelle.

Le dictionnaire devient ainsi un outil à l'intention d'usagers opérant en discours. Il sert de source de référence, de base de savoir pour l'acquisition de connaissances. Il s'inscrit dans un processus langagier d'énonciation (rédaction ou traduction) dont il marque une étape au lieu d'en être le point d'aboutissement. Cette conception de dictionnaire évolutif reconnaît le rôle terminologique du traducteur qui doit toujours garder une certaine marge de manoeuvre, une distance face aux usages terminologiques et une position critique lui permettant d'exercer pleinement sa créativité.

C'est devenu un lieu commun que de dire que le dictionnaire idéal n'existe pas, mais certains s'en approchent. Citons pour exemple le Dictionnaire de la comptabilité et de la gestion financière de $\mathrm{L}$. Ménard (1994) qui répond tant à l'exigence d'explication des concepts qu'à celle de fixation terminologique des usages (Dancette, 1994). Quoi qu'il en soit, rédiger un dictionnaire dans l'optique que nous proposons, en intégrant le processus d'énonciation, peut contribuer à renouveler nos conceptions sur la finalité des dictionnaires bilingues et sur leur usage. Nous espérons ainsi faire apparaître de nouveaux paradigmes de réflexion.

\section{Nature et dynamique de la langue du commerce de détail}

Chaque langage a des caractéristiques et des rythmes qui lui sont propres. Une évolution rapide du langage et son éclatement en de multiples sous-langages sont des traits qui, d'une façon générale, rendront la normalisation de ce langage difficile. Au contraire, la fixation et la concentration $d u$ vocabulaire en faciliteront la normalisation. 
Si nous définissons le langage du commerce de détail comme étant composé de tous les discours ayant trait au commerce de détail, de la petite épicerie de quartier (ou dépanneur) à l'hypermarché ou à la grande surface discompte de type Club Price (aujourd'hui Price-Costco) ou Wal-Mart, il faudra admettre que ce langage est difficilement normalisable en raison de plusieurs facteurs. Il est, en effet, traversé par des tendances à l'éclatement et subit une évolution extrêmement rapide entraînant, dans son sillage, un grand flou terminologique.

\subsection{Tendances à l'explosion terminologique (néologie et synonymie)}

Des néologismes apparaissent sans cesse sous l'effet de nombreux facteurs.

- Immensité et diversification croissante du domaine. Il suffit de penser à la place centrale qu'occupe le secteur du détail dans nos économies. Au Québec en 1993, il représente un chiffre d'afffaires de 42,5 milliards de dollars; il compte plus de 65000 points de vente et engage 220000 personnes (18\% des emplois). En France, l'appareil commercial comportait, en 1991, 523000 entreprises (presque un quart du total des entreprises) engageant $12 \%$ de la population active résidente.

Les intervenants agissent dans divers domaines: vente, gestion de magasin, techniques visuelles, transfert électronique de fonds, comptabilité, informatique, marchandisage, publicité, marketing, logistique, etc. - sont les faiseurs directs de la terminologie en évolution. Ajoutons à cela les urbanistes commerciaux, les architectes, les statisticiens, les législateurs, etc. Tous considèrent la réalité sous des angles différents et créent les termes dont ils ont besoin.

Donnons quelques exemples. Les opérations liées à la promotion immobilière ou aux stratégies marketing donnent naissance à un vocabulaire très diversifié. Citons en anglais les néologismes: mall, power center, off-price center, festival (ou entertainment) center, convenience center, etc. Les activités reliées au commerce électronique engendrent l'apparition de nouvelles appellations:échange de documents informatisés (EDI), transfert électronique de fonds (TEF), terminal de point de vente (TPV), code (â) barres, lecteur de code (à) barres. douchette, crayon-lecteur, pistolet laser, caisse enregistreuse 
électronique, lecteur de carte magnétique, saisie-clients (self scanning), etc. Celles liées à l'aménagement du magasin engendrent les termes linéaire, gondole, tête de gondole, frontal(e), etc. Les activités liées à la gestion du point de vente font apparaître les termes: surface brute commercialisable (ou surface brute de location), surface brute de vente, etc.

- Tendance à la profusion de termes. Même dans les secteurs traditionnels, moins touchés par les nouvelles techniques du commerce moderne et le gigantisme des structures, de très nombreux termes prolifèrent, cohabitent les uns à côté des autres, sans que les différences de sens ne semblent à l'origine motiver la présence de termes distincts.

Ainsi, en anglais, pour désigner le petit magasin de quartier, on trouve convenience store à côté de neigbo(u)rhood store, corner store, mom-and-pop store, bantam store, grocery store, etc. Comment devons-nous traiter ces termes dans le dictionnaire? Les faire tous apparaître comme vedettes ou les ranger comme synonymes, quitte à introduire des nuances ? Dans ce cas, sous quelle entrée vedette les placer ? Dans les cas où il est très difficile d'établir leur synonymie avec une grande certitude, jusqu'où est-il utile ou nécessaire de pousser l'étude des distinctions sémantiques, sachant que le terrain est très mouvant du fait de l'évolution rapide du domaine?

\subsection{Variabilité des concepts (polysémie, néosémie)}

- De vieilles notions prennent des sens nouveaux et, par conséquent, des sens nouveaux s'attachent à d'anciens termes. Prenons pour exemple le terme anglais discount store, souvent appelé en français magasin discount ou magasin de rabais, mais pour lequel nous proposons magasin discompte. Traditionnellement, il s'applique, entre autres, au magasin à prix unique du genre "Tout à un dollar», caractérisé par un assortiment limité et étroit, offrant, sur une petite surface située dans les vieux quartiers populaires, des produits bas de gamme ou de pacotille et attirant surtout une clientèle à faible revenu. Aujourd'hui, le même terme désigne aussi et parallèlement les très grandes surfaces discompte telles que Wal-Mart, Zeller et K-Mart. Situés généralement en périphérie des villes, ces magasins sont équipés des moyens techniques les plus modernes pour le paiement, la logistique et la gestion des 
ressources humaines. Contrairement aux magasins discompte traditionnels, ils offrent un large assortiment de produits de marque.

Pour des raisons que nous essaierons de cerner dans le point suivant, cette évolution des concepts, marquée par le fractionnement de la réalité et la diversification des notions, ne s'accompagne pas de la même évolution terminologique. À de nouvelles réalités correspondent de nouveaux termes (néologie). À plusieurs réalités correspond un seul terme (polysémie ou néosémie). Dans ces conditions, la description sémantique des termes peut devenir extrêmement complexe et longue, et faire l'objet d'un article qui ressemble plus à l'encyclopédie qu'au dictionnaire de langue.

- Des variantes culturelles. Le commerce de détail, attaché par définition aux clientèles locales, est nécessairement très touché par les différences régionales et culturelles. Ces différences expliquent qu'un même terme puisse désigner des réalités prenant des formes très diverses. L'exemple classique est celui de la pharmacie qui, en France, désigne généralement une surface de taille petite ou moyenne abritant l'officine du pharmacien et offrant principalement des médicaments et des produits à usage thérapeutique. La pharmacie nord-américaine, même si elle co-existe dans une version similaire à la pharmacie française, est généralement de taille beaucoup plus grande, et serait la conjonction, dans un magasin discompte, de la pharmacie et de la droguerie françaises, offrant aussi, bien que de façon marginale, de l'électronique, de l'alimentation, de la papeterie, etc., et même parfois les services d'un bureau de poste.

Dans le domaine du détail, en effet, nous avons affaire à des réalités conceptuelles et pragmatiques très variables. Avant même la désignation, les concepts peuvent être très différents d'une culture à l'autre. Toutefois, si nous les analysons, nous pouvons trouver des classèmes ou des lexèmes identiques ou proches, même s'il n'y a pas de correspondance parfaite entre les concepts sous-jacents.

On pourrait également mentionner l'exemple de l'hypermarché européen, grande surface à dominante alimentaire de type Continent, Cora ou Carrefour en France. Cette réalité n'a pas son équivalent en Amérique du Nord, malgré les tentatives récentes d'importation du 
concept. Le «hypermarket» américain est beaucoup plus influencé par les réalités du «discount» américain de genre Wal-Mart et Price-Costco.

Ainsi, nous nous retrouvons avec des termes qui, d'une culture à l'autre, revètent des sens différents. Ces particularités régionales rendent le transfert et la traduction difficiles, même quand les termes sont proches, et engendrent des faux amis ou des faux amis partiels. La non-univocité des équivalents explique sans doute que les spécialistes français du commerce de détail ne fassent guère d'effort pour franciser les termes d'origine américaine et préfèrent utiliser des emprunts (discount, off-price, power center, etc.). (Rappelons ici un vieux débat en linguistique fonctionnelle sur l'application aux emprunts de la notion d'économie. De plus, l'appellation anglo-américaine est investie d'un symbolisme, important en marketing, conférant à la réalité désignée des «qualités» américaines, le caractère moderne, le gigantisme, ou l'efficacité de la gestion.)

Dans un dictionnaire orienté sur les concepts, le plus important sera de faire comprendre en quoi ceux-ci diffèrent. Pour cela, le dictionnaire doit donner la description de ces différentes réalités avec les caractéristiques qui les distinguent. Là encore, l'aspect encyclopédique du dictionnaire est renforcé par rapport à un dictionnaire habituel.

\section{Degré embryonnaire de la recherche terminologique dans le commerce de détail}

Les degrés d'avancement des recherches lexicales et terminologiques dans un domaine donné déterminent la nature et la profondeur du travail du rédacteur de dictionnaire dans ce domaine. Ainsi, si nous prenons la finance ou la comptabilité, des dictionnaires existent depuis le début du siècle. Les nouvelles parutions se distinguent des précédentes par la mise à jour des informations, au mieux par une présentation de l'information tenant compte de la diversification du domaine et des spécialisations (ex. Dictionnaire économique et financier de la finance de la Banque de France, Glossaire de la Banque mondiale/The World Bank Glossary (1986), Glossaire anglais-français à l'usage des traducteurs du GATT (1984), The Wall Street Dictionary du New York Institute of Finance (1990), le Lexique des assurances, Banking Terminology de l'American Bankers Association (1989), etc.). 
En tout état de cause, les rédacteurs n'ont plus à partir de zéro ou très rarement, et n'ont plus à refaire l'organisation conceptuelle du domaine.

Dans le domaine du détail, au contraire, les outils terminologiques sont rares et assez pauvres car mal ciblés. Sans avoir la prétention de faire état d'un recensement exhaustif (et nous nous en excusons pour les ouvrages omis), nous avons trouvé en anglais le Dictionary of Retailing (Fairchild Publishers, 1985), le Dictionary of Buying and Merchandising (Krieger, 1990) et le Dictionary of Retailing (Baron, Davies \& Swindley, 1991); en français le Lexique du commerce moderne (Wellhoff, 1977) et le Dictionnaire commercial (Académie des sciences commerciales, 1987). Les ouvrages bilingues sont tous trop généraux. Nous avons relevé: Lexique du Marketing (Lacour, 1993), Dictionnairefrançais-anglais et anglais-françaisdumarketing(Chirouze et Metherell, 1990), Lexique de marketing et techniques commerciales (Brassart et Panazol, 1992), et un seul multilingue anglais/français/espagnol: Marketing trilingue (Chirouze, 1992).

Il est intéressant de s'interroger sur cette différence de traitement. Pourquoi la rechercheterminologique est-elle moins avancée ici qu'ailleurs ? Dans le domaine du détail, à l'inverse de la situation observée en finance ou en comptabilité, l'incitation à la normalisation $\mathrm{du}$ lexique par la voie d'ouvrages terminologiques est faible. Cette situation s'explique sans doute en partie par le fait que les diverses commissions et associations de commerce de détail ne jouent pas un rôle actif dans l'élaboration ou la normalisation de la terminologie. Ils ne publient ni recommandations terminologiques ni nomenclatures de termes. Les «faiseurs» de cette terminologie dont nous avons parlé plus haut - directeurs de marketing, directeurs de chaînes de distribution ou de centres commerciaux, promoteurs, vendeurs, etc. - forment, du moins sous cet aspect, un groupe disparate dont les usages langagiers ne sont contrôlés par aucune autorité linguistique. À leurs usages terminologiques s'ajoutent ceux des commentateurs externes tels que les universitaires et les journalistes, et des organismes gouvernementaux (StatCan, législateurs) selon les besoins de chacun.

Quand on voit des domaines entiers échapper à l'effort de normalisation terminologique, nous ne saurions qu'être d'accord avec Boulanger (1990:233) qui explique qu'il est irréaliste d'espérer trouver 
des répertoires complets de termes dans les dictionnaires bilingues: «En matière de terminologie et plus particulièrement dans les technolectes de pointe, la demande de mots dépasse de beaucoup les inventaires possibles.) Il donne un élément d'explication qui s'applique au français dans notre domaine: «À l'heure actuelle, le faible taux d'augmentation des dénominations endogènes entraîne des déficits lexicaux considérables, toujours accrus et nécessitant des efforts constants de créativité linguistique en vue de répondre aux défis technologiques et scientifiques majeurs qui révolutionnent inévitablement les sociétés contemporaines.»

Ces lacunes s'expliquent principalement par la vitesse à laquelle évolue la langue des affaires. Même les banques de terminologie informatisées, par ailleurs si précieuses, sont en retard sur la réalité: les contenus ne sont pas assez spécialisés; parmi les nombreux termes traités, les équivalences proposées sont souvent (et pour longtemps) «à vérifier» ou «à confirmen» et de nombreuses questions restent sans réponse. (On se reportera à l'analyse de Bessé (1992) pour l'analyse des banques de terminologie.)

Les retards terminologiques sur la réalité, qu'aucun dictionnaire ou banque de terminologie ne saurait vraiment rattraper, justifient que l'on cherche des solutions sous-optimales aux besoins des traducteurs. Si l'on ne peut offrir les répertoires de termes bilingues, au moins peut-on concevoir les outils qui permettront au traducteur de créer lui-même l'équivalent dont il a besoin en contexte.

\section{Besoins terminologiques du point de vue du traducteur}

\section{L'illusion ou le piège de l'équivalent presse-bouton.}

Snell-Hornby (1990) dresse un réquisitoire violent contre les dictionnaires à l'usage du traducteur. Elle voit une dichotomie entre les objectifs des lexicologues / lexicographes et les besoins des traducteurs. Il y a, dit-elle, un gouffre entre les deux, «(an) unbridgeable gap between the convictions of lexicologists and bilingual lexicographers on the one hand, and the needs of the translator as dictionary user on the other». Elle dénonce l'objectif fallacieux des «immediately insertable equivalents» que cherchent à atteindre les terminologues. 
Parce que le sens est «dynamique», qu'il s'habille en discours de nuances insaisissables hors contexte, Snell-Hornby remet en cause un postulat essentiel qui sous-tend non seulement les travaux des terminologues mais aussi les méthodes de travail de nombreux traducteurs débutants: l'équivalent n'est pas nécessairement ce dont a le plus besoin le traducteur.

Nous sommes, au moins en partie, d'accord avec ce raisonnement qui représente, pourrait-on dire, un choc épistémologique. Il faut en effet admettre que de nombreux dictionnaires sont peu utiles pour le traducteur professionnel, ou, pire, qu'ils sont des sources d'erreur. Pour ne donner qu'un exemple, mentionnons un dictionnaire des affaires, publié par une maison de dictionnaires très connue. Il donne pour le terme anglais investor deux équivalents: en premier actionnaire et en second investisseur; ou pour investment, une dizaine d'équivalents français sans indiquer aucune distinction de sens. Or un «investisseur» n'est pas nécessairement «actionnaire», et les termes mise de fonds, placement, investissement, etc. ne sont pas synonymes. Un traducteur non averti ou manquant de connaissances en finance n'arrivera pas à décider si les termes proposés fonctionnent comme équivalents adéquats dans le texte qu'il est en train de traduire car le dictionnaire bilingue ne donne pas les traits sémantiques qui permettraient de les distinguer les uns des autres.

"On ne trouve jamais rien dans les dictionnaires!»

Dans le domaine du détail, c'est plutôt l'absence des termes qui risque le plus de décourager le traducteur lorsqu'il consulte le dictionnaire. Des termes techniques comme big-box retailer, power center, category killer, off-price, etc. ne se trouvent ni dans le dictionnaire ni dans la plupart des banques de terminologie. La première difficulté pour le traducteur sera de reconnaître que ces expressions sont des syntagmes lexicalisés. Faute de quoi, il pourrait y voir des métaphores ne relevant que d'un idiolecte et les traduire librement. Par contre, s'il passe ce premier écueil, il devra trouver les moyens de poursuivre sa recherche documentaire et terminologique. Il ne pourra alors chercher le sens de ces termes que dans les revues spécialisées ou dans les manuels de cours de gestion. S'ils ne sont pas définis dans ces sources, il devra en inférer le sens des contextes dans lesquels ces termes paraissent et le vérifier 
auprès des experts du domaine. L'équivalent linguistique qu'il retiendra dans sa traduction (si ce n'est un emprunt) pourra ainsi faire l'objet de recherches interminables... peu rentables, si le traducteur est payé au mot.

\section{Changement de cap?}

Il faut admettre cette limite théorique: étant donné la nature du domaine et la rapidité à laquelle il évolue, aucun dictionnaire ne pourra jamais prétendre à l'exhaustivité et à l'actualité terminologiques. Dans ces conditions, les terminologues doivent-ils changer d'orientation et rechercher la richesse conceptuelle plutôt que la quantité de termes traités ? La description des qualités recherchées dans les articles de dictionnaire nous permettra d'analyser ce que le traducteur pourrait y gagner.

\section{Présentation du dictionnaire}

Nous reprenons le postulat énoncé plus haut, à savoir que les dictionnaires seraient beaucoup plus utiles et plus fiables pour la traduction s'ils contenaient une composante conceptuelle riche et structurée. En terminologie appliquée, cette exigence se traduit-elle par des caractéristiques originales ? On admettra, bien sûr, que l'intégration des connaissances conceptuelles a toujours été le point de départ du travail du terminologue. Toutefois, la sélection des entrées (macrostructure) et leur présentation (microstructure) reflètent assez rarement le travail d'acquisition, de regroupement, de classification des connaissances ou à des degrés très variables d'explicitation.

Le dictionnaire du commerce de détail que nous sommes en train d'élaborer se distingue des autres non pas tant par une caractéristique foncièrement nouvelle mais par une priorité particulière accordée au concept. Nous décrirons dans ce qui suit les différentes rubriques composant un article de dictionnaire.

\subsection{Divers types d'information}

Comme nous le disions en introduction, notre ouvrage intègre des renseignements d'ordre linguistique et d'ordre encyclopédique, qui tous 
reçoivent un traitement approfondi et intégré. Par ces divers types d'information, c'est la démarche cognitive même qui est visée, tant celle de l'équipe de rédaction du dictionnaire - qui se reflète de façon manifeste dans l'ouvrage - que celle de l'usager potentiel. Nous illustrons notre propos avec l'exemple de l'hypermarché. (Les termes apparaissant en caractères gras correspondent à des entrées de dictionnaire.)

\subsubsection{Connaissances linguistiques}

Nous distinguons les connaissances sémantiques des connaissances purement lexicales. Les connaissances sémantiques sont consignées dans la description du terme par ses principaux traits sémantiques:

Hypermarché $=$ Grande surface discompte à dominante alimentaire offrant en libre-service un assortiment très large de produits de grande consommation.

Les connaissances lexicales se rattachent aux connaissances sur le mot dans un contexte structural:

Le mot hypermarché est né, dans les années soixante, du besoin de désigner les "grands" supermarchés européens. On trouve aussi dans la presse spécialisée francophone la troncation hyper(synonyme), avec comme forme du pluriel hyper ou hypers.

Les connaissances interlinguistiques entrent dans cette catégorie:

En anglais, les termes hypermarket, super supermarket, supercenter et combination store sont les équivalents de hypermarché et de hyper.

\subsubsection{Connaissances encyclopédiques}

Nous distinguons, dans cette catégorie, (a) les connaissances concernant l'objet:

De manière générale, l'hypermarché est installé en zone périphérique ou suburbaine et dispose d'une grande aire de stationnement avec station-service; il joue fréquemment le rôle de locomotive dans les centres commerciaux. 
(b) les connaissances concernant l'origine de l'objet et son évolution:

Né en France en 1963, l'hypermarché est relativement nouveau en Amérique du Nord, où les secteurs alimentaire et non alimentaire restent fortement cloisonnés. Cependant, sous l'impulsion du géant américain Wal-Mart, un «hypermarché à l'américaine» émerge aux États-Unis.

(c) les connaissances concernant les relations internotionnelles:

L'hypermarché français, qui occupe une surface supérieure à 5000 $\mathrm{m}^{2}$, se distingue du supermarché de taille inférieure. Selon le Dictionnaire commercial (1987, p. 321), le terme très grand supermarché (TGS) (synonyme hypérette), est employé pour désigner l'hypermarché de 2500 à $5000 \mathrm{~m}^{2}$.

Aux États-Unis, le hypermarket est un concept à rapprocher du combination store (magasin combiné) par son assortiment très large (articles alimentaires, marchandises générales et services) et par la taille (entre $13000 \mathrm{~m}^{2}$ et $24000 \mathrm{~m}^{2}$ ). Il s'oppose au supermarket à dominance alimentaire et au superstore (supermagasin), spécialisé soit dans l'alimentaire soit dans une autre catégorie de produits.

Les liens notionnels sont, dans cette conception de la terminologie, aussi importants en tant qu'éléments porteurs d'information que les notions elles-mêmes en tant qu'unités spécifiques de connaissance. L'objectif recherché est de faciliter l'organisation et la classification des connaissances, et non pas seulement la simple désignation des notions dans les deux langues. Cependant, l'exemple présenté ici montre la difficulté à opposer clairement les concepts car les traits définitoires varient d'une région à l'autre et d'une époque à l'autre. (Nous nous sommes limitée ci-dessusà montrer les oppositions entre les notions américaines et françaises; des nuances seraient à ajouter si l'on considérait les concepts britanniques, canadiens, allemands, etc.)

Les connaissances concernant les variantes culturelles apparaissent souvent dans les relations internotionnelles, mais peuvent aussi être soulignées:

Le concept d'hypermarchén'existe pas au Québec. Le consommateur québécois qui en veut une idée claire devrait imaginer la combinaison d'un Canadian Tire, des rayons de vêtements d'un Sears, d'une succursale de la S.A.Q. et d'un marché d'alimentation Provigo ou Métro, ce qui en fait correspondrait à un «combination store». 
Des exemples sont donnés pour mieux illustrer les différences culturelles: American Fare, Wal-Mart Supercenter (défini comme "combination store») pour l'Amérique du Nord; Carrefour, Casino, Continent, Mammouth pour la France, Continente et Alcampo pour l'Espagne, définis comme «hypermarchés».

\subsection{Un réseau de connaissances}

Ainsi, à travers toutes ces catégories d'information, on cherche à définir avec le plus de précision et de rigueur possible les relations sémantiques de synonymie et d'antonymie et les relations hiérarchiques et associatives qui existent entre les termes. De ce fait, l'article de dictionnaire introduit l'usager dans le réseau complexe de connaissances attachées au concept, aux termes qui l'expriment dans chacune des deux langues, ainsi qu'aux relations qui lient ce concept aux concepts voisins par quelque critère que ce soit (ex.: taille, assortiment de produits, stratégie marketing, mode de propriété, etc.).

Il est important d'insister sur le processus de différenciation des concepts, le propre du travail du terminologue. C'est le classement des concepts par traits définitoires qui fait apparaître les relations générique/spécifique, partie/tout, et autres, et qui permet de mettre en évidence la multidimensionnalité conceptuelle. Par exemple, parmi les points de vente, certaines entités pourront correspondre à de multiples définitions sans que ces termes les qualifiant ne soient synonymes. Ainsi les magasins IKEA ou Toys «R» Us sont classés, dans la presse spécialisée, comme «big-box stores» (grandes surfaces discompte), "category killers» (grandes surfaces spécialisées ou méga-magasins spécialisés), «super-specialists» (superspécialistes), «superstores» (supermagasins), "discounters» (magasins discompte), «retail chains» (chaînes de magasins ou succursalistes). Dans cette série lexicale, les génériques discounter et big box store, très proches l'un de l'autre par certains aspects, ne peuvent cependant être donnés comme synonymes. Ils relèvent en effet de plans différents: la politique de prix pour le premier (la pratique du discompte) et, pour le second, la gestion des marchandises et la grande taille du magasin. De même, les termes category killer et superspecialist sont à rapprocher par leur assortiment très spécialisé; category killer et superstore, eux, sont à rapprocher par leur politique discompte et par leur taille, même si le category killer est une grande surface spécialisée de taille supérieure au superstore. Quant 
à retail chain, complètement à part dans la série, le terme relève du mode d'exploitation ou du mode de propriété de l'entreprise de vente au détail.

Au niveau de la démarche intellectuelle, la mise en évidence du processus cognitif au stade de l'élaboration du dictionnaire est à rapprocher de celle de l'usager du dictionnaire qui, lui aussi, a besoin de comprendre l'organisation conceptuelle du domaine dont relève son texte. Mais, à la différence du terminologue, le traducteur n'a pas nécessairement besoin de toute la gradation des termes à l'intérieur d'une série lexicale ni de toutes les relations. En fonction des éléments pertinents du discours, il aura soit à réduire l'arbre du domaine aux éléments qui l'intéressent (stratégie de réduction) soit à sélectionner et à simplifier les opérations de construction de l'arbre du domaine (stratégie de sélection). L'arbre du domaine présenté dans le dictionnaire doit donc être assez souple pour que le traducteur trouve le terme précis en faisant lui-même sa propre analyse. Toutefois, c'est par l'explicitation de la démarche de classification des concepts que l'ouvrage devient un outil de documentation essentiel au travail du traducteur.

\subsubsection{Explicitation des opérations de regroupement, de classement et de} catégorisation des concepts et des termes

À part les exceptions, dans la plupart des dictionnaires bilingues, ces opérations ne sont pas spécialement mises en lumière. Quand elles le sont, c'est de façon limitée, dans le meilleur des cas, par l'indication de l'appartenance du terme à un sous-domaine (ex.: «broker 1» commerce; «broker 2» valeurs mobilières; «broker 4» finance); par un système élémentaire de renvois (ex.: «broker 1» comparer avec "agent», «dealer 1»; voir aussi «intermediary", et l'indications des relations de synonymie (ex.: «broker 4» syn. «foreign exchange dealen», «foreign exchange trader»). (Les exemples ci-dessus sont extraits du Dictionnaire de la comptabilité et de la gestion financière (Ménard, 1994).)

Idéalement, le dictionnaire du commerce de détail devrait faire apparaître l'organisation conceptuelle globale du domaine. Si l'on sait que le commerce de détail est principalement structuré sur deux axes, à savoir le type d'établissement (taille, juridiction, statut) et la filière de 
produits (ex. "vêtement pour femme», «équipement du foyer», etc.), on pourrait espérer trouver dans le dictionnaire tout le vocabulaire concernant ces deux axes ainsi que toutes les relations sémantiques liant les termes les uns aux autres.

Notons cependant que les difficultés rencontrées dans cette tentative de structuration notionnelle du domaine nous donnent à penser qu'il est pratiquement impossible de présenter un système conceptuel qui rende compte du domaine d'une manière parfaitement cohérente, concrète et quelque peu durable.

Toutefois, cet idéal répond à un besoin souvent souligné par les traductologues. Dans le domaine économique, Gerzymisch-Arbogast (1989, p. 187) souligne que l'information relative aux relations paradigmatiques est indispensable pour minimiser ce que l'auteur appelle «the vagueness» du langage économique. L'indétermination des notions est source, dit l'auteur, des principales difficultés de traduction dans ce domaine.

Si le traducteur généraliste ou débutant ne peut espérer avoir en mémoire l'organisation conceptuelle des nombreux champs de connaissances auxquels les textes à traduire renvoient, il a besoin au moins d'ouvrages qui présentent les concepts, ne serait-ce que succinctement. Le traducteur devrait pouvoir y puiser ce que de Beaugrande et Dressler définissent comme «a configuration of knowledge that can be recovered or activated with more or less unity and consistency in the mind» (cité par Bühler, 1992, p. 424).

Une autre citation précise cette pensée:

La combinaison d'unités d'information et de connaissances hétérogènes nécessite, comme dans le cas des matériels et des logiciels, le recours à ce qu'on appelle des interfaces. C'est là que la terminologie a un rôle à jouer: on peut la considérer comme l'interface de l'intelligence humaine: elle rend possible la réalisation de liens notionnels entre les unités isolées, liens nécessaires à la production de connaissances. (Galinski et Nedobity, 1990, p. 56)

Et les auteurs d'ajouter: "Qu'une certaine dose de normalisation soit indispensable au bon fonctionnement des interfaces apparaîtra comme 
l'évidence à tous ceux qui ont l'habitude de manipuler des informations.» C'est cette conception de la terminologie qui nous paraît la plus utile en traduction.

Néanmoins, un ouvrage terminologique plus axé sur la description notionnelle que sur la «prescription» terminologique et l'«univocité» des équivalences aura plus de chance qu'un autre de résister à l'épreuve du temps et de la variabilité culturelle et linguistique. Établir une terminologie, c'est faciliter l'échange d'informations, par définitions communément acceptéeset partagéespar un ensemble d'usagers.

Pour tendre vers cet objectif, notre ouvrage comprendra, outre les entrées de dictionnaire, une introduction d'une vingtaine de pages sur le commerce de détail et des index des termes anglais et français permettant une consultation croisée. Tous les termes relatifs aux mots vedette, apparaissant dans les articles, figureront donc soit dans la vedette en tant que synonymes ou équivalents, soit dans l'index. Ces caractéristiques concernant la macrostructure et la microstructure permettent une consultation tant en extension qu'en intention. Nous pouvons ainsi parler d'une démarche cognitive globale, rendue accessible à l'utilisateur. Le dictionnaire devient alors un instrument de coopération entre le terminologue et l'usager. C'est peut-être dans cette voie que réside la solution au «unbrigeable gap».

\subsubsection{Appel à la collaboration du traducteur}

Inviter le traducteur à coopérer à l'établissement d'une terminologie en contexte et en évolution, plutôt qu'à recevoir l'information du dictionnaire de manière prescriptive et figée nous paraît être un objectif important pour plusieurs raisons dont certaines ont déjà été mentionnées. La variabilité culturelle et linguistique du vocabulaire, la non-univocité des équivalents, l'évolution rapide du vocabulaire font qu' aucun ouvrage ne peut résister à l'épreuve du temps. L'emploi de certains termes et expressions préconisé par les dictionnaires et les banques de terminologie est parfois arbitraire ou même criticable. Certains ont une durée de vie limitée comme l'a noté Wijnands dans son étude de la durabilité des néologismes (Wijnands, 1990). Certaines métaphores s'usent et deviennent clichés (ex.: boom, bubble economy, upbeat). Un 
emprunt, acceptable à un moment, est rejeté par certaines autorités quelques années plus tard (doit-on de ce fait remplacer marketing par mercatique?). Les «officialismes» (Boulanger, 1990, p. 238) reconnus dans une instance administrative sont rejetés ailleurs. Certaines propositions terminologiques faites par les terminologues ou les organismes de normalisation sont révisées en fonction de la complexification des notions.

Pour toutes ces raisons, le traducteur doit garder ses distances ou, du moins, rester critique. S'il doit savoir se conformer à la terminologie en usage (parfois consacrée par les textes de loi), le traducteur devra être souple car, dans les domaines du langage très liés aux aspects socio-culturels, les termes prennent des extensions de sens, subissent des changements d'usage, ou tombent en désuétude en fonction des reformulations de lois et réglements. Par exemple, en France, en vertu de la loi sur la réforme boursière du 22 janvier 1988, les charges d'agent de change sont remplacées par les sociétés de bourse. Ainsi, il n'est plus possible de traduire broker par agent de change pour décrire une réalité ultérieure à la date d'entrée en vigueur de la loi.

\section{Conclusion}

Arrivée au terme de cet article, nous espérons avoir montré qu'en traduction, domaine où le texte résulte d'un acte discursif unique, les voies de la recherche terminologique s'inscrivent dans une sémantique contrastive et une lexicologie fonctionnelle. Une telle approche offre l'avantage de faire apparaître non seulement les variantes notionnelles et linguistiques liées aux différences culturelles, mais aussi, même si nous en avons peu parlé, les particularités phraséologiques attachées à l'emploi des termes. Dans tous les cas où les sources terminologiques sont muettes, le traducteur ne pourra choisir ses équivalents qu'en fonction de sa compréhension du sujet. La place primordiale des connaissances thématiques dans le processus de traduction est encore une fois soulignée. En terminologie bilingue, le besoin de dictionnaire à cheval sur le répertoire de termes et l'encyclopédie est encore une fois affirmé. 
Jeanne Dancette: Département de linguistique et de traduction, Université de Montréal, CP 6128, succ. A, Montréal H3C $2 \mathrm{J7}$ (Québec)

\section{Références}

Académie des sciences commerciales(1987). Dictionnaire commercial. Paris, CILF.

BARON; J., Davies, B. \& Swindley. D. (1991). Dictionary of Retailing. Londres, McMillan.

BEAUGRANDE, R. (de) et W. Dressler (1981). Introduction to Textlinguistics. Londres, Longman.

BERNARD, M. (1992). Le commerce de détail et son milieu. Montréal, HEC.

BÉJOINT, H. (1990). «Monosemy and the Dictionary», Budalex '88 proceedings, T. Magay and J. Zigany, eds. Budapest, Akademia Kiado.

BÉLANGER, G. (1991). «Pour une nouvelle terminographie», Meta, XXXVI(1).

BOULANGER, J.-C. (1989). «Lexicographie et politique langagière: l'exemple français des avis officiels" in F.J. Hausman, O. Reichmann, H.E. Wiegand, L. Zgusta, dir. Wörterbücher, Dictionnaires, Encyclopédies, tome I. Berlin, N.Y, Walter de Gruyter.

(1990). «La création lexicale et la modernité», Le langage et l'homme, XXV(4).

BRASSART, U. et J. M. Panazol (1992). Lexique de marketing et techniques commerciales.

BÜHLER, H. (1992). "Of Terms and Texts», Terminologie et Traduction, II(3). Luxembourg, Commission des communautés européennes. 
BUSKIRK, R. H. et B. Buskirk (1978). Retailing. Toronto, McGraw Hill.

(1982). La gestion des commerces de détail. Toronto, McGraw Hill, trad. de Retailing (1978). Toronto, McGraw Hill.

CHANSOU, M. (1990). «Termes de la finance et de la Bourse, essai d'organisation d'une terminologie», Banque des mots, numéro spécial.

CHIROUZE, Y. (1992). Le marketing trilingue. Paris, Chotard.

CHIROUZE, Y. et V. Metherell (1990). Dictionnaire français-anglais et anglais-français du marketing. Paris, Chotard.

DANCETTE, J. (1992). «La complexité de la langue économique et commerciale au Québec», Terminologie et Traduction, 2/3, Luxembourg, Commission des Communautés européennes.

(1993). «Translating the Language of Business», in Edith F. Losa, dir. Proceedings, 34th Annual Conference of the American Translators Association. Medford, NJ, Learned Information, Inc.

(1994a). «A Dictionary of Retailing in the Making». Proceedings of the 35th Annual Conference of the American Translators Association.

(1994b). «Dans les pas du 'Sylvain': Le Dictionnaire de la comptabilité et de la gestion financière de L. Ménard, M. Arsenault et J.-F. Joly", Circuit,

DUPUIS, M. (1991). Marketing international de la distribution. Paris, Les éditions d'organisation.

FILSER, M. (1989). Canaux de distribution. Paris, Vuibert.

GAMBIER, Y. (1993). «Socioterminologie et phraséologie: pertinence théorique et méthodologique», Terminologie et Traduction, II(3), Luxembourg, Commission des Communautés européennes. 
GALINSKI, C et W. Nedoobity (1990). «Une banque de données terminologiques considérée comme outil de gestion», La banque des mots, IX(2).

GAULIN, M. \& al. (1993). Les commerces de détail. Marketing et gestion (adaptation of Mason, J. B. \& Mayer, M.L. Modern Retailing. Theory and Practice). Montréal, Gaétan Morin.

GERZYMISCH-ARBOGAST, H. (1989). «The Role of Sense Relations in Translating Vague Business and Economic Texts», in Snell-Hornby, M., E. Pohl et B. Bennani (1989) Translation and Lexicology.

GUÉVEL, Z. (1990). «Traduction et développement de la terminologie française dans le domaine des affaires», Meta, $\operatorname{XXV(2).~}$

KRIEGER (1990). Dictionary of Buying and Retailing.

LACOUR, J.-P. (1993). Lexique du marketing. Paris, Éd. Bertrand-Lacoste.

LYONS, J. (1970). Linguistique générale. Paris, Larousse.

MASON, J. B. et M. L. Mayer (1990). Modern Retailing, Theory and Practice. $5^{\mathrm{e}}$ édition, Homewood et Boston, B.B.I. Irwin.

MÉNARD, 1. (1994). Dictionnaire de la comptabilité et de la gestion financière. Toronto et Montréal, Institut canadien des comptables agréés.

REY-DEBOVE, J. (1971). Étude linguistique et sémiotique des dictionnaires français contemporains. La Haye, Mouton.

ROCHARD, M. (1992). «Terminologie et traduction», Commission des communautés européennes, II(3).

SAGER, J. C. (1990). A Practical Course in Terminology Processing. Amsterdam, John Benjamins.

(1993). Language Engineering and Translation. Amsterdam, John Benjamins. 
SAGER, J.C. et K. Kageura (1994-1995) "Concept Classes and Conceptual Structures: Their Role and Necessity in Terminology", Terminology and LSP Linguistics. Studies in Specialized Vocabularies and Texts. Actes de Langue française et de Linguistique (ALFA) 7/8.

SCHANK, R. et R. Abelson (1977). Scripts, Plans, Goals and Understanding. Hillsdale, N.J., Lawrence Erlbaum.

SNELL-HORNBY, M. (1988). Translation Studies, An Integrated Approach. Amsterdam, John Benjamins.

(1990). «Dynamics in Meaning as a Problem for Bilingual Lexicography», in J. Tomaszczyket B. Lewandowska-Tomaszczyk,dir., Meaning and Lexicography. Philadelphie, John Benjamins.

SNELL-HORNBY, M., E. Pohl et B. Bennani, dir. (1989). Translation and Lexicography. Philadelphia, John Benjamins.

VIGNY, J. (1990). La distribution, structures et techniques. Paris, Éditions Sirey.

WELLHOFF, A. (1977). Lexique du commerce moderne. Paris, Éditions d'organisation.

WIJNANDS, P. (1990). «Analyses néologiques à partir des mots nouveaux apparus depuis 1985», Banque des mots, numéro spécial.

RÉSUMÉ: Organisation conceptuelle du domaine et structure de dictionnaire - L'exemple du commerce de détail - Le présent article a pour objectif de présenter un dictionnaire spécialisé bilingue (anglaisfrançais) original. Il est conçu pour répondre aux besoins des traducteurs et les stratégies lexicographiques mises en œuvre s'orientent autour de trois axes: richesse des propositions d'équivalent, intention et extension des définitions, explicitation des relations sémantiques (ou internotionnelles). L'article propose une discussion de ces axes en fonction de plusieurs paramètres liés au domaine de spécialité: nature et dynamique de la langue commerciale dans le domaine de la distribution, degré d'avancement de la recherche terminologique dans 
ce domaine, besoin des traducteurs dans cette spécialité. Les grandes lignes théoriques de l'article s'articulent autour de la mise en évidence des structures de connaissances propres au domaine et de la priorité accordée à la démarche cognitive des traducteurs (qui opèrent en discours plutôt qu'en langue).

ABSTRACT: Conceptual Organisation and Dictionary Structure in Specialized Fields: The Example of Retailing - The aim of this article is to present an original bilingual (English-French) specialized dictionary. Designed to meet the needs of translators, its lexicographic strategies revolve around three elements: richness of equivalents, intension and extension of definitions, clarification of semantic relations. The article discusses these elements focusing on several parameters related to the field of specialization: nature (and dynamics) of the language of retailing, degree of advancement of terminological research, and the needs of translators in this field. Our theoretical proposals highlight the structures of knowledge in the specialized field and give priority to the cognitive process involved in translation (on the level of discourse rather than language). 